\title{
Public Transport Scheme Optimized by Election Campaign Algorithm and Extension Theory
}

\author{
Qinghua Xie ${ }^{1, a,{ }^{*}}$, Xiangwei Zhang ${ }^{1, b}$, Wenge $L^{1, c}$ and Siyuan Cheng ${ }^{1, d}$ \\ ${ }^{1}$ School of Electromechanical Engineering, Guangdong University of Technology, Guangzhou china \\ axqhcool@163.com, bxwzhang@gdut.edu.cn, 'Ivwenge@163.com, dimdesign@gdut.edu.cn \\ ${ }^{*}$ Corresponding author
}

Keywords: Public transport scheme; Optimization; Election campaign algorithm; Extension theory

\begin{abstract}
In order to improve the demand of rational layout of public transport system which include buses and metro in cities, this paper propose the method of optimization with Election Campaign Algorithm and extension theory to plan the public transport scheme. Based on the extension method of decision choose problem, the evaluation model is constructed by extension multi-factor evaluation method. It alters the qualitative index into quantitative index and accurately reflect service quality of urban public transit. The experimental results show that the method can really solve the actual problem of city public transport service.
\end{abstract}

\section{Introduction}

Metro and bus are the most important component of city public traffic system, and metro traffic associated with conventional public transit network reasonable, is a fundamental step to improve the city public transportation service efficiency [1]. The conventional bus transit network is composed of the conventional bus transit routes which serve the passenger flow between the metros. Every connecting bus line of metro station run in accordance with the specific frequency [2,3]. So, the problem of the feeder line network is to make sure that the urban bus transit route scheme is composed of three parts, which are the connection of the metro station, the line, and the operating frequency.

Extenics is a cross discipline founded by Professor Cai [4]. It proposes math language to research things of development [5]. With extension innovation rules and methods, it resolves mainly the conflict problems [6]. Extenics has been applied to the economic, management, decision-making, information technology, new product design and intelligent control.

Election campaign algorithm (ECA) is a new optimization algorithm simulating election process [7]. Election candidates always pursue the maximum support from voters by means of various election behaviors. They get the overview of their support from the voters according to the sample survey, and then decide what to do on the next move. Solution space is imagined as voters and current solutions are imagined as candidates in ECA. The function value of a feasible solution is named as the prestige of a voter and the function value of a current solution is named as the prestige of a candidate.

In ECA, the candidates influence the voters round them, the voter's support is in proportion to the distance between the candidates and the voters [8]. The voter will vote to the candidate which he pefer the most. The sum of location coordinates of every voters supported the candidate powered by its contribution is a new location coordinates, which is named support focus, it is the next position of the candidate. Such computational cycle is done continually until a candidate finds the position of the highest support, which is the global solution of the optimization problems.

In this paper, we use election campaign algorithm and extension theory to optimize the public transport scheme. The optimization model of transit network is established based on the benefit of the passenger flow and the operator's benefit as the optimization objective. The author uses extension comprehensive evaluation method to evaluate the objective. 


\section{Optimization Model of Transit Line Network}

The minimize cost of passengers generalized travel and operator operating as the goal $[9,10]$, the objective function of public transport scheme can be calculated by Eq. 1 .

$$
\begin{aligned}
& \min Z=A+B+C \\
& A=\frac{\lambda_{T}}{v_{T}}\left(\sum_{d=I+1}^{I+J} \sum_{j=I+1}^{I+J} \sum_{i=1}^{I} P_{i d} Y_{i j} L_{j d}\right) \\
& B=B_{1}+B_{2} \\
& B_{1}=\lambda_{w}\left(\sum_{k=1}^{K} \frac{1}{2 f_{k}} \sum_{d=I+1}^{I+J} \sum_{i=1}^{I} \sum_{h=1}^{I+J} P_{i d} X_{i h k}\right) \\
& B_{2}=\frac{\lambda_{r}}{v_{B}} \sum_{k=1}^{K} L_{k} \sum_{d=I+1}^{I+J} P_{k d} \\
& C=2 \lambda_{b}\left(\sum_{k=1}^{K} f_{k} \sum_{h=1}^{I+J} \sum_{i=1}^{I} X_{i h k} L_{i h}\right)
\end{aligned}
$$

As is shown in Eq. 1, the optimization model is the sum of passenger's metro cost $A$, waiting cost $B_{1}$, ride cost $\mathrm{B} 2$ and public transport operation $\operatorname{cost} C$. Y is defined as the affiliation between the bus stations, and $X$ is defined as the affiliation between the bus stations and the metro line. $H$ is a collection of all the stations and parts of the bus station.

$$
\begin{aligned}
& Y_{i j}=\left\{\begin{array}{l}
1, \mathrm{i} \text { and } \mathrm{j} \text { are adjacent to each other } \\
i=1, \cdots, I ; j=I+1, \cdots, I+J \\
0, \text { others }
\end{array}\right. \\
& X_{i j}=\left\{\begin{array}{l}
1, \mathrm{i} \text { and } \mathrm{h} \text { are adjacent to each other in section } \mathrm{k} \\
i=1, \cdots, I+J ; j=1, \cdots, I+J ; k=1, \cdots, K \\
0, \text { others }
\end{array}\right. \\
& \sum_{i \notin H} \sum_{h \in H} \sum_{k=1}^{K} X_{i h k} \geq 1
\end{aligned}
$$

$Y_{i j}$ between line $\mathrm{k}$ and station $\mathrm{I}$, station $\mathrm{J}$ must meet the following constraints.

$$
\sum_{h=1}^{I+J} X_{i h k}+\sum_{p=1}^{I} X_{p j k}-Y_{i j} \leq 1, i=1, \cdots, I ; j=I+1, \cdots, I+J ; k=1, \cdots, K
$$

Bus operating frequency $f_{k}$ should meet the line requirements of the transport capacity. $C$ is the capacity of each bus; $\rho$ is the utilization of seats.

$$
\sum_{i=1}^{I} \sum_{d=I+1}^{I+J} P_{i d} \sum_{h=1}^{I+J} X_{i h k} \leq f_{k} C_{\rho} ; k=1, \cdots, K
$$

Bus line frequency is limited by the total mileage of transit vehicles during the operating time. $t$ is the average readiness and reentry time of the bus during time period $T . N$ is the number of operation bus during time period $T$.

$$
\sum_{k=1}^{K} f_{k} \sum_{i=1}^{I} \sum_{h=1}^{I+J} L_{i h} X_{i h k} \leq \frac{1}{2} N v_{B}(T-t)
$$

\section{Correlation Function}

In this paper, we use the extension elementary correlation function as the measure tool of the object function.

If set $x$ to any point on the real axis, $X_{0}=\langle a, b\rangle$ is any interval on the real field [6].

$$
\rho\left(x, X_{0}\right)=\left|x-\frac{a+b}{2}\right|-\frac{b-a}{2}
$$

The distance between point $x$ and interval $X_{0}$ is calculated by Eq. 13 . 


\section{Election Campaign Algorithm}

Framework of the election campaign algorithm is described as the follows:

Set the Algorithm Parameters. Main parameters of Election campaign algorithm are:

- The number of candidates.

- The number of voters.

- The number of floating voters.

- Target accuracy.

Generate the Candidates and Calculate the Prestige of Them. Generated the define number of candidates in feasible solution field on the uniform distribution. Use the objective function to calculate the prestige of each candidate.

Generate the Voters. The uniform distribution is employed to generate the voters in feasible solution field.

Compute the Investigate Mean Square of Candidates. Higher prestige of a candidate, smaller the mean square deviation of local voters, so that ECA is able to converge to local optimization solution rapidly and steadily. The following formula is used to describe the relation of the prestige and the mean square deviation of a candidate.

$$
\sigma_{C_{i}}=\frac{\left(P_{M a x}-P_{\mathrm{C}_{i}}\right)}{\left(P_{\text {Max }}-P_{\text {Min }}\right)}
$$

Where $\sigma_{C_{i}}$ represents the mean square deviation of candidate $C_{i} ; P_{M a x}$ and $P_{M i n}$ are the maximum and minimum prestige of candidates.

Next step, voters are generated around each candidate on the normal distribution.

$$
N_{C_{i}}=\frac{\left(P_{M a x}-P_{\mathrm{C}_{i}}\right)}{\left(P_{\text {Max }}-P_{\text {Min }}\right)}\left(N_{V}-N_{F V}\right)
$$

$N_{C_{i}}$ Is the voter number of candidate $C_{i}, N_{V}$ is the number of all voters, $N_{F V}$ is the number of floating voters. Floating voters are generated on the uniform distribution. Here, it is supposed that candidate's effect on voters declined linearly.

Calculate the Supported of the Voters. Candidates can influence the voters within their effect range, the effect on voter $V_{j}$ from candidate $C_{i}$ is

$$
F_{C_{i} V_{j}}=\frac{D_{C_{i} M a x}-D_{C_{i} V_{j}}}{D_{C_{i} \operatorname{Max}}} P_{C_{i}}
$$

$F_{C_{i} V_{j}}$ is the effect on voter $V_{j}$ form candidate $C_{i}, V_{j}$ is the global or local voter. Here, it is supposed that candidate's effect on voters declined linearly.

Then Compute the prestige of voters by means of objective function.

The support of a voter is proportional to his prestige, and then the proportional constant will be reduced, so the prestige of a voter can used to denote the support of a voter directly. A voter may be influenced by several candidates; the voter should distribute his support to candidates proportionally on the magnitude of effect from candidate to voter. The support from voter $V_{j}$ to candidate $C_{i}$ is

$$
S_{C_{i} V_{j}}=\frac{F_{C_{i} V_{j}}}{\sum_{i=1}^{m} \max \left(F_{C_{1} V_{j}}, F_{C_{2} V_{j}}, \cdots, F_{C_{i} V_{j}}, \cdots F_{C_{m} V_{j}}\right)} P_{V_{j}}
$$

$S_{C_{i} V_{j}}$ represents the support from the voter $V_{j}$ to candidate $C_{i}$.

Find the Support Focus of Candidates. A new position coordinate will achieve by means of summing the products of the support from the voters to the candidate and the position coordinate of the voters. It is named the support focus. 


$$
x_{C_{i}}^{*}=\frac{\sum_{j=1}^{n} S_{C_{i} V_{j}} x_{V_{j}}}{\sum_{j=1}^{n} \max \left(S_{C_{i} V_{1}}, S_{C_{i} V_{2}}, \cdots, S_{C_{i} V_{j}}, \cdots S_{C_{i} V_{n}}\right)}
$$

$x_{C_{i}}^{*}$ is the support focus of the candidate $C_{i}$. The support focus of a candidate is obtained by investigating, which depends on those voters whose distances to the candidate are nearer and the prestige is higher relatively. The next post of the candidate should be the support focus, where the candidate will have the higher support.

Calculate the Prestige of the Candidates and Compare the Prestige of the Voters with the Candidates. In order to jump out of local optimization solution and increase search rate, the prestige of candidates are compared to that of voters, if the prestige of a voter is higher than that of a candidate, the voter with higher prestige will substitute for the candidate and the that candidate of lower prestige will be eliminated in election.

Check Whether the Condition is Reach. Check whether the condition is reach, otherwise return to start step to execute the period. Here, the condition could be the max cycle.

$$
\left|Z-\frac{\min \left(x^{*}\right)+\max \left(x^{*}\right)}{2}\right| \leq \frac{\max \left(x^{*}\right)-\min \left(x^{*}\right)}{2}
$$

The total cost $Z$ has many possible results. In these result, we use the extension elementary correlation function to design which result is accepted. When the max cycle reach, the computation stop. The results left at the last could be the most possible results, the minimum of them will be the approximate best design we could get.

\section{Sample of Detecting Maximum Inscribed Rectangle}

Using Election campaign algorithm, the number of candidates define as 5, the number of voters is 10 , the number of floating voters is 5 , and the target accuracy is set as $1 \%$. The experiment get the maximum inscribed rectangle of the stone slab, as is show in Fig. 3, it prove the feasibility of the algorithm. Parameters in the model are shown in Tab. 1.

Table 1 Model parameters

\begin{tabular}{|c|c|c|c|c|c|c|c|c|c|c|c|c|}
\hline [parameter] & $C$ & $\rho$ & $t$ & $N$ & $\lambda_{w}$ & $\lambda_{r}$ & $\lambda_{T}$ & $\lambda_{j}$ & $\lambda_{B}$ & $V_{B}$ & $V_{T}$ & $\lambda$ \\
\hline [unit] & $\mathrm{p}$ & & $\mathrm{h}$ & & $/\left(\mathrm{p}^{*} \mathrm{~h}\right)$ & $/\left(\mathrm{p}^{*} \mathrm{~h}\right)$ & $/\left(\mathrm{p}^{*} \mathrm{~h}\right)$ & $/ \mathrm{p}$ & $/\left(\mathrm{p}^{*} \mathrm{~km}\right)$ & $\mathrm{km} / \mathrm{h}$ & $\mathrm{km} / \mathrm{h}$ & \\
\hline [value] & 200 & 1.2 & 0.15 & 100 & 22 & 16 & 15 & 0.3 & 18 & 20 & 40 & 0.001 \\
\hline
\end{tabular}

The experiment achieve the design of the public transport system model, it prove the feasibility of the algorithm.

\section{Conclusions}

In the model of public transport system, transfer fee is added to the passenger travel cost; so that the transfer networks of passenger travel cost is more comprehensive. According to the characteristic of the model, the election campaign algorithm and extension elementary correlation function are used. The evaluation function is introduced as a direct line connecting the line through the station and the cost; determine the line frequency considering the objective function and constraints. Example analysis shows that the optimization results are reasonable.

Election campaign algorithm has good performance to avoid the solution trapped in local optima. This is particularly important when dealing with highly multimodal functions. The extension evaluation model is the application of extension theory in the field of complex connected networks designing. For future work, extension theory will be used to solve more design and evaluation problem. 


\section{References}

[1] G. K. Kuah, J. Perl, Optimization of feeder bus routes and bus-stop spacing, Transport Engineering, 1988, 114(3), pp. 341-354.

[2] G. K. Kuah, J. Perl, The feeder-bus network-design problem, Journal of the Operational Research Society, 1989(40), pp. 751-767.

[3] C. L. Matins, M. V. Pato, Search strategies for the feeder bus network design problem, Europe Journal Operational Research, 1998(106), pp. 425-440.

[4] Wen Cai, Extension theory and its applications, Chinese Science Bulletin, 1999, 44(17), pp. 1538-1548.

[5] Yongquan $\mathrm{Yu}$, Haixia Peng, The principle of extension detecting with extension sets, Proc of International Conference on Computer, Communication and Control Technologies, Orlando, INTINST Informatics \& Systemics, 2003, pp. 113-118.

[6] M. H. Wang, Y. F. TSENG, A novel clustering algorithm based on the extension theory and genetic algorithm, Expert Systems with Applications, 2009,36(4), pp. 8269-8276.

[7] Wenge Lv, Qinghua Xie, Zhiyong Liu, Deyuan Li, et al., Verifying Election Campaign Optimization Algorithm by Several Benchmarking Functions, Lecture Notes in Computer Science, 6146 LNCS, 2, pp. 582-57.

[8] Wenge Lv, Chunhua He, DeyuanLi, et al., Election Campaign Optimization Algorithm. Procedia Computer Science, 1, pp. 1371-1380.

[9] S. N. Kuan, H. L. Ong, K. M. Ng, Applying metaheuristics to feeder bus network design problem, Asia-Pacific Journal of Operational Research, 2004, 21(4), pp. 543-560.

[10] S. N. Kuan, H. L. Ong, K. M. Ng, Solving the feeder bus net-work design problem by genetic algorithms and ant colony optimization, Advances in Engineering Software, 2006(37), pp. 351-359. 